\title{
Buonismo, buonista, falso buonismo e cattivismo
}

\section{Giuseppe Patota}

PUBBLICATO: 08 LUGLIO 2019

\section{Quesito:}

"Nei vocabolari che ho in casa non ho trovato i termini buonismo e buonista. Li trovo raccapriccianti, non fosse che per i contesti in cui vengono proferiti (di solito nei confronti dell'accoglienza ai migranti). Qual è la loro prima attestazione?", ci ha chiesto una lettrice. "Mi chiedo spesso perché sarebbe tanto negativo essere buonisti, quale sarebbe la differenza dall'essere semplicemente buoni e perché il suo contrario cattivismo non sia altrettanto diffuso", ha domandato una seconda lettrice, alla quale ha fatto eco una terza, che ha chiesto: "Si potrebbe dire cattivista?". C'è poi chi ci ha chiesto se, visto che buonismo è sinonimo di 'falsa bontà', riteniamo l'espressione falso o finto buonismo un pleonasmo intenzionale o un errore.

\section{Buonismo, buonista, falso buonismo e cattivismo}

P rima di rispondere, mi permetto di dire che condivido, delle prime due domande, la premessa che le incornicia: credo, infatti, che buonismo ebuonista siano due parole-alibi, usate soprattutto per negare la possibilità stessa che qualcuno possa essere buono e impegnarsi per un mondo con meno ingiustizie.

La mia opinione personale, però, prescinde totalmente dalla risposta che ho il compito di dare in quanto linguista, che comincia da qui.

Il nome buonismo e il nome e aggettivo buonista entrano in circolazione e si affermano - segnatamente, nel linguaggio giornalistico - negli ultimi anni del secolo scorso.

Degli ormai molti vocabolari che hanno accolto entrambi i termini a partire dal ı996, il primo ad aver ascritto correttamente al 1993 la loro comparsa è il Sabatini-Coletti 2008.

Se la storia di queste due parole comincia nel I993, la loro preistoria inizia diversi decenni prima: nel I956 per buonismo e nel 1969 per buonista. Dico preistoria perché i contesti in cui le due parole compaiono in un tempo cosi remoto ne mostrano la natura del tutto occasionale e la non riproducibilità: nel merito, chiedo alle lettrici e ai lettori di aver pazienza, perché darò notizie più particolareggiate in un articolo che sto tuttora scrivendo insieme a Dalila Bachis.

Quando cominciarono a essere usate nei primi anni novanta del Novecento, le parole buonismo e buonista indicavano, rispettivamente, un 'atteggiamento di apertura e di tolleranza nei confronti dell'avversario politico' e qualcuno o qualcosa 'che mostra un atteggiamento di apertura e di tolleranza nei confronti dell'avversario politico'. Negli ultimi anni del secolo scorso e nei primi di questo secolo, però, è andato affermandosi un nuovo significato, che nell'uso ha ormai completamente soppiantato il precedente: esso interpreta il buonismo come un'ostentazione di falsa bontà, in particolare nei confronti dei migranti, di minoranze etniche e talvolta anche di chi delinque' e qualifica buonista qualcuno (o qualcosa) 'che ostenta falsa bontà nei confronti dei migranti, di minoranze etniche e talvolta anche di chi delinque'. Falsa bontà, ho scritto: a mio avviso l'espressione falso buonismo, più che un errore, è un pleonasmo (preterintenzionale, non volontario) in cui l'aggiunta 
della parola falso è determinata dal fatto che il buonismo comporta (secondo chi ama usare il termine) falsità. Cattivismo, il suo contrario, esiste, e alcuni dizionari lo hanno accolto. L'ultimo Zingarelli (Zingarelli 2019) lo descrive nel modo che segue: 'Nel linguaggio giornalistico, atteggiamento di chi usa intenzionalmente toni aspri o esibisce cattivi sentimenti'. Veramente, il nuovo significato che buonismo ha ormai assunto imporrebbe di aggiornare anche quello di cattivismo, sporadicamente (e polemicamente) adoperato, nella stampa e nella rete, da chi sopporta poco l'escalation della parola buonismo. Conclusa la risposta destinata agli utenti, propongo, di cattivismo, la definizione che segue:

cattivismo m. (plur. -i) ostentazione di cattivi sentimenti, di intolleranza e di malevolenza portate al massimo grado verso altri esseri umani diversi per etnì, nazionalità, fede religiosa, identità sessuale, bassa o bassissima condizione socioeconomica, praticata in particolare da uomini politici o anche da giornalisti che ne condividono le posizioni.

Nota bibliografica:

- Michele A. Cortelazzo, Le parole della neopolitica - Buonista, (o5 aprile 20r9, http://www.treccani.it/magazine/lingua_italiana/articoli/parole/Neopoliticarr.html).

- Maria Vittoria Dell'Anna, Pierpaolo Lala, Mi consenta un girotondo. Lingua e lessico nella Seconda Repubblica, Galatina, Congedo, 2004.

- Federico Faloppa, Per un linguaggio non razzista, in Marco Aime, Guido Barbujani, Clelia Bartoli, Federico Faloppa, Contro il razzismo. Quattro ragionamenti, a cura di Marco Aime, Torino, Einaudi, 2016, pp. 69-I24.

\section{Cita come:}

Giuseppe Patota, Buonismo, buonista, falso buonismo e cattivismo, "Italiano digitale", 2019, X, 2019/3 (luglio-settembre)

DOI: $10.35948 / 2532-9006 / 2020.3192$

Copyright 2019 Accademia della Crusca

Pubblicato con licenza creative commons CC BY-NC-ND 